\title{
Free-living Capybaras (Hydrochoerus hydrochaeris) in an Urban Area in Brazil - Biochemical and Hematological Parameters
}

\author{
Raissa Brauner Kamla Vieira ${ }_{\odot}^{1}$, Vinícius Silva Rodrigues ${ }_{\oplus}^{2}$, Laís Miguel Rezende ${ }_{\oplus}^{2}$, \\ Maria Marlene Martins ${ }^{2}$, Caroline Lopes Queiroz ${ }^{2}$, Matias Juan Pablo Szabó ${ }^{2}$, \\ Nádia Regina Pereira Almosny ${ }^{3}{ }^{3} \&$ Nathalie Costa da Cunha ${ }_{\odot}^{1}$
}

\begin{abstract}
Background: Capybaras have found favorable conditions for survival and reproduction in green urban environments. In recent years, the population of these large rodents has been increasingly abundant in several brazilian cities such as Uberlândia, a municipality of the southeastern region with a Cerrado biome. Capybaras are important in the Brazilian Spotted Fever epidemiological chain, by amplifying infection rates of the vector population. However, knowledge of this host's physiology is scarce. Thus, the aim of this work was to describe hematological and biochemical parameters of free-living capybaras groups in urbanized areas in the city of Uberlândia, Minas Gerais State, Brazil.

Materials, Methods \& Results: Capybaras were captured in 4 different locations of Uberlândia city, Minas Gerais state, including 1 Condominium (P1), 1 Private Market Garden (P2), 1 Private Club (P3) and 1 Municipal Park (P4). The animals were baited into an octagonal iron corral and chemically contained with anesthetic darts. After sedated, blood was collected from the femoral vein in tubes with and without EDTA. Biochemical evaluation, hematological analysis with differential leukocyte counts and search for Dirofilaria sp. were done. The blood count and biochemistry values obtained from animals of different ages, sex and sectors (P1, P2, P3 and P4) were submitted to the Shapiro-Wilk normality test, considering 95\% significance. Values that had a normal distribution were subjected to ANOVA tests followed by Student's $t$-test. Values that did not follow normality were submitted to the Kruskal-Wallis test, to obtain a $P$-value, with a significance level of 95\%. A total of 19 capybaras were captured: 4 in P1, 6 in P2, 4 in P3 and 5 in P4. From the 19 animals, 13 were females (68.42\%) and 6 were males (31.57\%), 12 adults (63.15\%) and 7 juveniles (36.84\%). Apart from occasional skin scars and moderate to intense Ambyomma spp. tick infestations, all captured animals were healthy on a broad examination. From 5 animals captured in $\mathrm{P} 4$, despite the use of anticoagulant, blood from 4 animals clotted fast. No microfilariae were found in the thick drop test in any of the 19 animals sampled, and in 2 adult female capybaras captured in P1, Kurloff cells were observed. Hematological and biochemical values presented no major differences when comparing sex and age. Nevertheless, differences in liver and kidney profile were observed between the capybara groups, including ALT, alkaline phosphorus, BUN and creatinine.

Discussion: Blood from 4 animals clotted fast, despite the use of EDTA tubes. Blood clotting of samples with anticoagulant in this work could be associated with some physiological features inherent to capybaras. Many attempts were required to obtain enough blood from each individual due to the rapid hemostasis, what come in accordance with reports in literature. Kurloff cells were observed in 2 adult female capybaras captured in P1, which can be found in peripheral blood of female rodents during follicular phase of estrous cycle. Hematological and biochemical values differences in liver enzymes such as ALT and alkaline phosphorus, and kidney profile enzymes including BUN and creatinine could be associated to capture stress or dietetic variation between groups. Despite statistical relevant, the values were still in accordance with other works, although comparisons should be done with caution since various environments exert a diverse array of stimulus upon the animals such as parasitic, infective, stress, nutritional, social and undoubtedly blood parameters mirror them. In conclusion, this work contributes to the standardization of free-living capybaras' physiological parameters in urban areas.
\end{abstract}

Keywords: capybaras, physiological parameters, enzymatic parameters, green urban environment, capture stress, fauna, biomes.

DOI: 10.22456/1679-9216.114437

Accepted: 12 0ctober 2021

Published: 22 November 2021

Received: 24 June 2021

${ }^{1}$ Department of Veterinary Collective and Public Health \& ${ }^{3}$ Department of Veterinary Clinical Pathology, Faculty of Veterinary Medicine, Fluminense Federal University (UFF), Niterói, RJ, Brazil. ${ }^{2}$ Laboratory of Ixodology, Faculty of Veterinary Medicine, Federal University of Uberlândia (UFU), Uberlândia, MG, Brazil. CORRESPONDENCE: R.B.K. Vieira [raissabrauner@gmail.com]. Department of Veterinary Collective and Public Health UFF. Rua Vital Brazil Filho s/n. CEP 24230-340 Niterói, RJ, Brazil. 


\section{INTRODUCTION}

Alteration of natural biomes by agriculture and urbanization, also alters animal populations [2]. The original fauna is replaced by animal species that best adapt to the new landscape. In this context, the capybara (Hydrochoerus hydrochaeris) considered the largest rodent in the world, with feeding plasticity adapted well to human modified landscapes [6]. It is also associated with the transmission of the zoonotic agent Rickettsia rickettsii, a highly lethal bacteria that causes Brazilian Spotted Fever. This disease is transmitted to humans by the tick Amblyomma sculptum previously infected by capybaras, one of its primary host species [11].

The capybara is semi-aquatic and needs water to mate, thermoregulate and for protection against predators [1]. Thus, green anthropized areas with water may provide adequate environment for it [13]. The city of Uberlândia, in the Cerrado biome, and the second in population size in Minas Gerais (MG) state, witnessed an increase in the population of capybaras and large groups are seen in municipal parks, townhouses and riparian vegetation by the Uberabinha River $[15,16,17]$. In the anthropized environment, the availability and abundance of resources reflects on their body condition. Such areas tend to offer greater availability of food [2] and, changes in enzymatic parameters can occur in animals under different climatic and resources conditions [18].

The objective of this work is to determine hematological and biochemical parameters of 4 different groups of capybaras in the city of Uberlândia-MG, contributing to the knowledge about the parameters of these anthropized rodents within diverse environment.

\section{MATERIALS AND METHODS}

\section{Study locations}

The study was conducted from December 2018 until July 2020 in 4 different locations within the urban area Uberlândia, Minas Gerais. This city is in the Mesoregion of the Triângulo Mineiro / Alto Paranaíba southeastern Brazil, with approximately 700,000 inhabitants and, so far, not considered endemic for spotted fever. It is inserted within the Cerrado biome and has a tropical climate with mild winter and a hot and rainy summer [10,17]. Diet of the animals varied according to the place where they lived, ranging from grasses, vegetables, dry corn and Panicum and Pennisetum grass. Coordinates, characteristics of the location and available food are described in Table 1.

\section{Method of capturing and collecting material}

Capybaras were baited with corn and sugar cane, into an octagonal iron corral (walls with $1.50 \mathrm{~m}^{2}$ each) with a guillotine door. Animal access was monitored by a camera trap ${ }^{1}$. Baiting period varied according to each location, on average 2 months. Captured animals were chemically contained with anesthetic darts containing $3.0 \mathrm{mg} / \mathrm{kg}$ of xylazine $2 \%^{2}$ and $10 \mathrm{mg} / \mathrm{kg}$ of cetamine $10 \%{ }^{2}$, with the weight estimated by their size, according to IBAMA [9].

With animals under anesthesia, a mean of 23 $\mathrm{mL}$ of blood was collected by puncture of the femoral vein in VHS tubes without and with EDTA anticoagulant. Except for cubs due to anesthetic risk, all captured animals were included in the research, regardless of sex or age. Fur from the dorsal cervical region of captured capybara was cut to avoid double sampling if captured again (approximately $20 \mathrm{~cm}$ length), and long-term marking with a small perforation in the ears. After full recovery from anesthesia capybaras were released at the same site of capture.

\section{Sample processing}

Blood samples were submitted to biochemical and hematological analysis performed at the Veterinary Clinical Pathology Laboratory, on ChemWell ${ }^{3}$ multichannel automatic analyzer equipment and on a pocH automatic analyzer $-100 \mathrm{iV}$ Diff ${ }^{4}$, respectively. Differential leukocyte counts were performed on blood smears stained by the May-Grünwald-Giemsa method, taking into account the presence of heterophils in capybaras blood [8]. A thick drop test was also performed to detect microfilariae under an optical microscope.

\section{Evaluation of parameters}

The following biochemical parameters were evaluated: Albumin (g/dL), Triglycerides (mg/dL), Total Proteins (g/dL), Calcium (mg/dL), Phosphorus (mg/ $\mathrm{dL})$, Cholesterol (mg/dL), Aspartate Aminotransferase (AST U/L), Gamma Glutamyl Transferase (GGT U/L), Alkaline Phosphatase (FA - U/L), Alanine Aminotransferase (ALT - U/L), BUN (mg/dL), Creatinine (mg/dL).

The hematological parameters evaluated were Leukocytes (x10 $)$, Red blood cells (RBC 10\% $/ \mathrm{mm}^{3}$ ), 
Hemoglobin (HGB g/dL), Hematocrit (HCT\%), Mean Corpuscular Volume (MCV fL), Mean Cell Hemoglobin (MCH pg), Mean Corpuscular Hemoglobin Concentration (MCHC g/dL) and platelets $\left(\times 10^{3}\right)$.

\section{Statistical analysis}

The blood count and biochemistry values obtained from animals of different ages, sex and sectors
(P1, P2, P3 and P4) were submitted to the ShapiroWilk normality test, considering $95 \%$ significance. Values that had a normal distribution were subjected to ANOVA tests followed by Student's t-test. Values that did not follow normality were submitted to the Kruskal-Wallis test, to obtain a $P$-value, with a significance level of $95 \%$. The tests were performed using the BioEstat program 5 .

Table 1. Capybara capture location and its environment, city of Uberlândia-MG.

\begin{tabular}{|c|c|c|}
\hline Location & Environment & Capybaras sampled \\
\hline $\begin{array}{c}\text { Condominium - P1 } \\
\left(18^{\circ} 53^{\prime} 12^{\prime \prime} \mathrm{S} 48^{\circ} 21^{\prime} 08^{\prime \prime} \mathrm{W}\right)\end{array}$ & $\begin{array}{l}\text { Fenced horizontal residential condominium, with a dam, } \\
\text { surrounded by grass which served as food for capybaras. }\end{array}$ & 3 adult females, 1 juvenile female. \\
\hline $\begin{array}{l}\text { Private Social Club - P3 } \\
\left(18^{\circ} 56^{\prime} 06^{\prime \prime} \mathrm{S} 48^{\circ} 17^{\prime} 34^{\prime \prime} \mathrm{W}\right)\end{array}$ & $\begin{array}{l}\text { Private sport and social club by the Uberabinha River, with } \\
\text { remnants of natural vegetation, bamboo, and grassy areas. Animals } \\
\text { were fed by local vegetation in and outside the Club. }\end{array}$ & 3 adult males and 1 adult female. \\
\hline $\begin{array}{c}\text { Municipal Park - P4 } \\
\left(18^{\circ} 56^{\prime} 22^{\prime \prime} \mathrm{S} 48^{\circ} 14^{\prime} 19^{\prime \prime} \mathrm{W}\right)\end{array}$ & $\begin{array}{l}\text { Fenced Park located in an urban area, Presence of small dam, non- } \\
\text { native Cerrado trees and hydrophytes, and garbage (clothes and } \\
\text { furniture); food provided by the residents and workers of the park. }\end{array}$ & $\begin{array}{l}2 \text { adult females, } 2 \text { juvenile females, } \\
\qquad 1 \text { juvenile male. }\end{array}$ \\
\hline
\end{tabular}

\section{RESULTS}

A total of 19 capybaras were captured: 4 in P1, 6 in P2, 4 in P3 and 5 in P4. From the 19 animals, 13 were females $(68.42 \%)$ and 6 were males $(31.57 \%)$, 12 adults $(63.15 \%)$ and 7 juveniles $(36.84 \%)$. Apart from occasional skin scars and moderate to intense Ambyomma spp. tick infestations, all captured animals were healthy on a broad examination. From 5 animals captured in P4, despite the use of anticoagulant, blood from 4 animals clotted fast and were inappropriate for blood analysis and therefore statistical evaluation of some parameters. No microfilariae were found in the thick drop test in any of the 19 animals sampled. In 2 adult female capybaras captured in P1, Kurloff cells were observed. Blood with anticoagulant from another individual from the same capture site presented a clot after venipuncture, despite the normal platelet counts.
Comparison of the hematological and biochemical parameters according to gender and age did not reveal major significant differences, except for platelets and basophil counts between males and females (Table 2), and phosphorus levels between adults and juveniles (Table 3).

Comparisons of the hematological parameters among the capybara groups from three collection sites revealed significant differences of MCHC between animals captured in P1 and P2 (Table 4).

Circulating monocyte numbers differed significantly between capybaras from P2 and P3 (Table 5 ) and the remaining blood parameters were similar among the 3 groups of capybaras.

Biochemical parameters from blood revealed several significant differences among capybaras from different capture sites (albumin, ALT, FA, BUN and creatinine) (Table 6). 
R.B.K.Vieira, V.S. Rodrigues, L.M. Rezende, et al. 2021. Free-living Capybaras (Hydrochoerus hydrochaeris) in an Urban Area in Brazil Biochemical and Hematological Parameters.

Table 2. Mean, standard deviation and amplitude of comparative hematological parameters between male and female capybaras (Hydrochoerus hydrochaeris) and between young and adults in the city of Uberlândia-MG.

\begin{tabular}{|c|c|c|c|c|}
\hline \multirow{2}{*}{ Parameter } & \multicolumn{2}{|c|}{ Sex } & \multicolumn{2}{|c|}{ Age } \\
\hline & Females & Males & Adults & Juveniles \\
\hline $\mathrm{RBC}\left(\mathrm{x} 10^{6} / \mathrm{mm}^{3}\right)$ & $\begin{array}{l}2.90 \pm 0.22 \\
(2.72-3.14)\end{array}$ & $\begin{array}{l}2.88 \pm 0.3 \\
(2.5-3.49)\end{array}$ & $\begin{array}{l}2.92 \pm 0.25 \\
(2.63-3.49)\end{array}$ & $\begin{array}{c}2.92 \pm 0.23 \\
(3.3-2.97)\end{array}$ \\
\hline HGB $(g \%)$ & $\begin{array}{c}12.89 \pm 0.94 \\
(12.2-14.2)\end{array}$ & $\begin{array}{c}12.72 \pm 1.71 \\
(11-14.8)\end{array}$ & $\begin{array}{l}12.95 \pm 1 \\
(12-14.8)\end{array}$ & $\begin{array}{c}12.82 \pm 0.96 \\
(11-13.9)\end{array}$ \\
\hline $\operatorname{HCT}(\%)$ & $\begin{array}{l}38.2 \pm 2.14 \\
(36.7-40.5)\end{array}$ & $\begin{array}{c}38.47 \pm 3.26 \\
(33-43.8)\end{array}$ & $\begin{array}{c}38.55 \pm 2.61 \\
(36.4-43.8)\end{array}$ & $\begin{array}{c}38.22 \pm 2.28 \\
(33-38.9)\end{array}$ \\
\hline MCV (fL) & $\begin{array}{c}124.54 \pm 24.53 \\
(124.6-139.2)\end{array}$ & $\begin{array}{c}122.8 \pm 30.83 \\
(41.8-132)\end{array}$ & $\begin{array}{c}125.08 \pm 24.4 \\
(41.8-140.7)\end{array}$ & $\begin{array}{c}122.56 \pm 26 \\
(117.9-134.7)\end{array}$ \\
\hline $\mathrm{MCH}(\mathrm{pg})$ & $\begin{array}{c}44.37 \pm 1.78 \\
(42.4-47.3)\end{array}$ & $\begin{array}{c}44.26 \pm 1.83 \\
(41.9-45.6)\end{array}$ & $\begin{array}{l}44.4 \pm 1.75 \\
(41.9-47.9)\end{array}$ & $\begin{array}{c}43.84 \pm 1.24 \\
(42.1-44.3)\end{array}$ \\
\hline $\mathrm{MCHC}(\mathrm{g} / \mathrm{dL})$ & $\begin{array}{c}33.73 \pm 1.35 \\
(31.4-35.8)\end{array}$ & $\begin{array}{c}33.06 \pm 1 \\
(33.2-34.4)\end{array}$ & $\begin{array}{l}33.6 \pm 1.23 \\
(31.4-35.8)\end{array}$ & $\begin{array}{c}33.53 \pm 1.31 \\
(32-33.3)\end{array}$ \\
\hline Platelets $\left(\mathrm{x} 10^{3}\right)$ & $\begin{array}{c}85.78 \pm 55.12^{\mathrm{a}} \\
\quad(42-213)\end{array}$ & $\begin{array}{c}63.88 \pm 31.36^{\mathrm{b}} \\
\quad(22-70)\end{array}$ & $\begin{array}{c}78.5 \pm 49.28 \\
\quad(24-213)\end{array}$ & $\begin{array}{c}75 \pm 44.66 \\
(22-172)\end{array}$ \\
\hline Leucocytes $\left(\mathrm{x} 10^{3}\right)$ & $\begin{array}{c}7.47 \pm 1.64 \\
(4-10.3)\end{array}$ & $\begin{array}{c}7.96 \pm 1.71 \\
(8.1-9.9)\end{array}$ & $\begin{array}{c}7.68 \pm 1.76 \\
(4.8-9.9)\end{array}$ & $\begin{array}{c}7.48 \pm 1.78 \\
(4-10.3)\end{array}$ \\
\hline Heterophils (\%) & $\begin{array}{c}46.35 \pm 10.35 \\
(22-55)\end{array}$ & $\begin{array}{c}45.5 \pm 7.76 \\
\quad(36-57)\end{array}$ & $\begin{array}{c}45.21 \pm 9.7 \\
(36-57)\end{array}$ & $\begin{array}{c}46 \pm 112 \\
(22-59)\end{array}$ \\
\hline Eosinophils (\%) & $\begin{array}{c}14.5 \pm 3.91 \\
(8-20)\end{array}$ & $\begin{array}{c}14.5 \pm 4.3 \\
(9-21)\end{array}$ & $\begin{array}{c}14.78 \pm 3.78 \\
(8-17)\end{array}$ & $\begin{array}{c}14.33 \pm 4.18 \\
(11-21)\end{array}$ \\
\hline Basophils (\%) & $\begin{array}{c}0.63 \pm 0.61^{\mathrm{a}} \\
(0-1)\end{array}$ & $\begin{array}{c}0.66 \pm 0.7^{\mathrm{b}} \\
\quad(1-2)\end{array}$ & $\begin{array}{c}1 \pm 0.6 \\
(0-2)\end{array}$ & $\begin{array}{c}0.83 \pm 0.57 \\
(0-1)\end{array}$ \\
\hline Monocytes (\%) & $\begin{array}{c}3.21 \pm 1.85 \\
(2-5)\end{array}$ & $\begin{array}{c}3.5 \pm 2.3 \\
(1-7)\end{array}$ & $\begin{array}{c}3.42 \pm 2.17 \\
(1-7)\end{array}$ & $\begin{array}{c}3 \pm 1.85 \\
(0-4)\end{array}$ \\
\hline Lymphocytes (\%) & $\begin{array}{c}34.85 \pm 10.84 \\
(25-59)\end{array}$ & $\begin{array}{c}35.2 \pm 9.29 \\
(19-45)\end{array}$ & $\begin{array}{c}35.5 \pm 10.91 \\
(19-45)\end{array}$ & $\begin{array}{c}35.58 \pm 12 \\
(25-59)\end{array}$ \\
\hline
\end{tabular}

Different letters on the line indicate that there is a statistical difference, with a $95 \%$ significance level.

Table 3. Mean and standard deviation of comparative biochemical parameters between male and female capybaras (Hydrochoerus hydrochaeris) and between young and adults in the city of Uberlândia-MG.

\begin{tabular}{|c|c|c|c|c|}
\hline \multirow{2}{*}{ Parameter } & \multicolumn{2}{|c|}{ Mean \pm SD } & \multicolumn{2}{|c|}{ Mean \pm SD } \\
\hline & Females & Males & Adults & Juveniles \\
\hline Albumin (g/dL) & $\begin{array}{c}1.90 \pm 0.3 \\
(1.37-2.55)\end{array}$ & $\begin{array}{c}1.90 \pm 0.3 \\
(1.5-2.3)\end{array}$ & $\begin{array}{l}1.87 \pm 0.32 \\
(1.37-2.55)\end{array}$ & $\begin{array}{c}1.89 \pm 0.3 \\
(1.56-2.06)\end{array}$ \\
\hline Total Proteins $(\mathrm{g} / \mathrm{dL})$ & $\begin{array}{l}5.63 \pm 0.58 \\
(4.92-6.79)\end{array}$ & $\begin{array}{l}5.82 \pm 0.61 \\
(5.06-6.89)\end{array}$ & $\begin{array}{l}5.75 \pm 0.55 \\
(4.96-6.89)\end{array}$ & $\begin{array}{l}5.61 \pm 0.59 \\
(4.92-5.88)\end{array}$ \\
\hline Creatinine (mg/dL) & $\begin{array}{l}1.01 \pm 0.25 \\
(0.66-1.57)\end{array}$ & $\begin{array}{l}0.93 \pm 0.2 \\
(0.7-1.13)\end{array}$ & $\begin{array}{c}1 \pm 0.27 \\
(0.67-1.57)\end{array}$ & $\begin{array}{c}1 \pm 0.25 \\
(0.7-1.12)\end{array}$ \\
\hline BUN (mg/dL) & $\begin{array}{c}21.46 \pm 9.94 \\
(4.5-32.2)\end{array}$ & $\begin{array}{c}24.3 \pm 10.49 \\
(27.1-39.3)\end{array}$ & $\begin{array}{c}22.31 \pm 10.15 \\
(4.5-39.3)\end{array}$ & $\begin{array}{c}21.8 \pm 10.12 \\
(11.1-30.4)\end{array}$ \\
\hline $\operatorname{AST}(\mathrm{U} / \mathrm{L})$ & $\begin{array}{c}40.89 \pm 15.35 \\
(24-66)\end{array}$ & $\begin{array}{c}39.41 \pm 17.6 \\
(30-82)\end{array}$ & $\begin{array}{c}41.31 \pm 16.53 \\
(24-82)\end{array}$ & $\begin{array}{c}41.05 \pm 15.78 \\
(30-47)\end{array}$ \\
\hline $\operatorname{ALT}(\mathrm{U} / \mathrm{L})$ & $\begin{array}{c}64.05 \pm 24.99 \\
(9-111)\end{array}$ & $\begin{array}{c}64.16 \pm 22.3 \\
(67-102)\end{array}$ & $\begin{array}{c}70.18 \pm 21.10 \\
(32-111)\end{array}$ & $\begin{array}{c}62.72 \pm 25 \\
(9-78)\end{array}$ \\
\hline GGT (U/L) & $\begin{array}{c}7.5 \pm 2.98 \\
(-1.2-12)\end{array}$ & $\begin{array}{c}8.08 \pm 2.35 \\
(5-13)\end{array}$ & $\begin{array}{c}7.53 \pm 3.18 \\
(5.1-9)\end{array}$ & $\begin{array}{c}7.6 \pm 3 \\
(-1.2-13)\end{array}$ \\
\hline $\mathrm{FA}(/ \mathrm{L})$ & $\begin{array}{c}177,84 \pm 105,52 \\
(74-386)\end{array}$ & $\begin{array}{c}133,33 \pm 66,02 \\
(94-130)\end{array}$ & $\begin{array}{c}189,93 \pm 111,12 \\
(74-386)\end{array}$ & $\begin{array}{c}166,27 \pm 95,38 \\
(77-298)\end{array}$ \\
\hline Calcium (mg/dL) & $\begin{array}{l}10.19 \pm 1.28 \\
(8.53-12.45)\end{array}$ & $\begin{array}{c}9.74 \pm 1.22 \\
(8-11.36)\end{array}$ & $\begin{array}{c}10.25 \pm 1.2 \\
(8.53-12.45)\end{array}$ & $\begin{array}{c}10.18 \pm 1.31 \\
(8-11.89)\end{array}$ \\
\hline Phosphorus (mg/dL) & $\begin{array}{c}5.47 \pm 1.06 \\
(4-7.32)\end{array}$ & $\begin{array}{l}5.21 \pm 0.94 \\
(4.17-6.98)\end{array}$ & $\begin{array}{c}5.19 \pm 0.9^{\mathrm{a}} \\
(4-7.07)\end{array}$ & $\begin{array}{c}5.45 \pm 1.09^{b} \\
(5.17-7.32)\end{array}$ \\
\hline Triglycerides (mg/dL) & $\begin{array}{c}33.63 \pm 29.3 \\
(8-135)\end{array}$ & $\begin{array}{c}26.66 \pm 12.84 \\
(14-58)\end{array}$ & $\begin{array}{c}32.93 \pm 31.34 \\
\quad(11-135)\end{array}$ & $\begin{array}{c}33.33 \pm 30.12 \\
(8-72)\end{array}$ \\
\hline Cholesterol uiform (mg/dL) & $\begin{array}{c}33.52 \pm 11.02 \\
(19-52)\end{array}$ & $\begin{array}{c}32.91 \pm 9.21 \\
(18-48)\end{array}$ & $\begin{array}{c}30.87 \pm 9.78 \\
(19-52)\end{array}$ & $\begin{array}{c}33.72 \pm 11.31 \\
(18-52)\end{array}$ \\
\hline
\end{tabular}

Different letters on the line indicate that there is a statistical difference, with a 95\% significance level. 
Table 4. Average, standard deviation and amplitude of hematological parameters of capybaras (Hydrochoerus hydrochaeris) captured in different urban areas of Uberlândia-MG.

\begin{tabular}{cccc}
\hline Parameter/unit & P1 & P2 & P3 \\
\hline \multirow{2}{*}{ RBC $\left(\mathrm{x} 10^{6} / \mathrm{mm}^{3}\right)$} & $3.03 \pm 0.08$ & $2.76 \pm 0.16$ & $2.9 \pm 0.32$ \\
& $(2.93-3.14)$ & $(2.5-2.97)$ & $(2.63-3.49)$ \\
HGB $(\mathrm{g} \%)$ & $13.55 \pm 0.55$ & $12.23 \pm 0.62$ & $13 \pm 1.12$ \\
& $(12.9-14.2)$ & $(11-12.8)$ & $(12.1-14.8)$ \\
HCT $(\%)$ & $38.82 \pm 0.84$ & $37.6 \pm 2.46$ & $39.7 \pm 3.1$ \\
& $(37.7-40)$ & $(33-40.5)$ & $(36.4-43.8)$ \\
MCV $(\mathrm{fL})$ & $128.2 \pm 2.69$ & $119.2 \pm 34.70$ & $130.7 \pm 6.1$ \\
& $(124.6-132.1)$ & $(41.8-139.2)$ & $44.3 \pm 2.38$ \\
MCH $(\mathrm{pg})$ & $44.72 \pm 1.78$ & $44.25 \pm 0.82$ & $(41.9-47.9)$ \\
& $(42.4-47.3)$ & $(43.1-45.6)$ & $33.87 \pm 0.44^{\mathrm{ab}}$ \\
MCHC $(\mathrm{g} / \mathrm{dL})$ & $34.87 \pm 0.78^{\mathrm{a}}$ & $32.56 \pm 0.74^{\mathrm{b}}$ & $(33.2-34.4)$ \\
& $(34-35.8)$ & $(31.4-33.3)$ & $59.75 \pm 23$ \\
Platelets $\left(\mathrm{x} 10^{3}\right)$ & $102.5 \pm 67.5$ & $75 \pm 35.2$ & $(24-87)$ \\
& $(42-213)$ & $(22-123)$ & $(7.2-.9 .9)$ \\
\hline
\end{tabular}

Different letters on the line indicate that there is a statistical difference, with a 95\% significance level.

Table 5. Mean of percentage, standard deviation and amplitude referring to the differential count of capybaras (Hydrochoerus hydrochaeris) captured in different areas of the city of Uberlândia-MG.

\begin{tabular}{cccc}
\hline Parameter/unit $(\%)$ & P1 & P2 & P3 \\
\hline \multirow{2}{*}{ Heterophils } & $42.25 \pm 12.57$ & $46 \pm 8.08$ & $47 \pm 6.16$ \\
& $(22-55)$ & $(35-55)$ & $(41-57)$ \\
Eosinophils & $14 \pm 2.54$ & $16.16 \pm 4.29$ & $13.5 \pm 2.69$ \\
& $(10-17)$ & $(8-21)$ & $(9-16)$ \\
Monocytes & $3.25 \pm 1.08^{\mathrm{ab}}$ & $1.83 \pm 1.21^{\mathrm{a}}$ & $6.0 \pm 1.22^{\mathrm{b}}$ \\
& $(2-5)$ & $(0-4)$ & $(4-7)$ \\
Basophils & $0.75 \pm 0.43$ & $0.5 \pm 0.5$ & $1 \pm 0.7$ \\
& $(0-1)$ & $(0-1)$ & $32.5 \pm 8.95$ \\
Lymphocytes & $38.75 \pm 13.27$ & $35.33 \pm 8.61$ & $(19-44)$ \\
& $(25-59)$ & $(25-47)$ &
\end{tabular}

Different letters on the line indicate that there is a statistical difference, with a 95\% significance level.

\section{DISCUSSION}

In this study blood parameters of free-living capybaras from a major urban area in Minas Gerais State, Brazil was provided. It also displayed minor differences that may exist among capybara groups from the same urban area.

All sampled animals, despite tick infestations were healthy, therefore these results may be used as reference for free-living capybaras from anthropized and urban areas. In fact, the categorization of capybaras into captive or free-living and further into free-living from pristine or anthropized either urban or rural areas is relevant in the evaluation of various physiological parameters of capybaras. These various environments exert a diverse array of stimulus upon the animals such as parasitic, infective, stress, nutritional, social and undoubtedly blood parameters mirror them. Therefore, comparisons should be done with caution.

The hematological values of the red series obtained in the present study generally matched previous ones with capybaras with some differences [5]. The referred work exhibited higher values of circulating leukocytes (segmented, eosinophils and monocytes) in free-living animals in relation to those found in the animals of the present study. In that work, high number of circulating white cells were attributed to parasitological diseases found in those animals living in the Atlantic rainforest biome. Although parasitic diseases were not specifically addressed in our work, and despite Amblyomma spp. tick infestations of all 19 captured animals, they all displayed healthy appearance that may explain lower leukocytes counts. 
Table 6. Average, standard deviation and amplitude of the hematological parameters of capybaras (Hydrochoerus hydrochaeris) captured in different areas of the city of Uberlândia-MG.

\begin{tabular}{|c|c|c|c|c|}
\hline Parameter/unit & P1 & $\mathrm{P} 2$ & P3 & P4 \\
\hline Albumin (g/dL) & $\begin{array}{c}1.88 \pm 0.07^{\mathrm{ab}} \\
(1.79-1.99)\end{array}$ & $\begin{array}{l}1.56 \pm 0.12^{\mathrm{a}} \\
(1.37-1.73)\end{array}$ & $\begin{array}{c}2.07 \pm 0.15^{\mathrm{b}} \\
(1.91-2.3)\end{array}$ & $\begin{array}{l}2.17 \pm 0.2^{b} \\
(2.01-2.55)\end{array}$ \\
\hline Total Proteins $(\mathrm{g} / \mathrm{dL})$ & $\begin{array}{l}5.41 \pm 0.33 \\
(5.03-5.95)\end{array}$ & $\begin{array}{c}5.54 \pm 0.3 \\
(4.96-5.88)\end{array}$ & $\begin{array}{l}6.39 \pm 0.53 \\
(5.53-6.89)\end{array}$ & $\begin{array}{c}5.30 \pm 0.4 \\
(4.92-6.06)\end{array}$ \\
\hline BUN (mg/dL) & $\begin{array}{c}15.85 \pm 4.92^{\mathrm{ab}} \\
(9.6-23.4)\end{array}$ & $\begin{array}{c}23.5 \pm 5.84^{\mathrm{ab}} \\
(14.4-30.4)\end{array}$ & $\begin{array}{l}34.3 \pm 3.45^{\mathrm{a}} \\
(30.2-39.3)\end{array}$ & $\begin{array}{c}13.26 \pm 7.5^{\mathrm{b}} \\
(4.5-27.3)\end{array}$ \\
\hline Creatinine $(\mathrm{mg} / \mathrm{dL})$ & $\begin{array}{c}1.31 \pm 0.2^{\mathrm{a}} \\
(1.03-1.57)\end{array}$ & $\begin{array}{c}0.74 \pm 0.07^{\mathrm{b}} \\
(0.66-0.88)\end{array}$ & $\begin{array}{c}0.98 \pm 0.09^{\mathrm{ab}} \\
(0.88-1.13)\end{array}$ & $\begin{array}{c}1.13 \pm 0.06^{\mathrm{b}} \\
(1.07-1.25)\end{array}$ \\
\hline Triglycerides (mg/dL) & $\begin{array}{c}48.25 \pm 51.5 \\
(8-135)\end{array}$ & $\begin{array}{c}33.3 \pm 19.6 \\
(19-72)\end{array}$ & $\begin{array}{c}23.5 \pm 6.1 \\
(14-31)\end{array}$ & $\begin{array}{c}30.4 \pm 14.4 \\
(17-58)\end{array}$ \\
\hline Cholesterol (mg/dL) & $\begin{array}{c}30 \pm 13.47 \\
(19-52)\end{array}$ & $\begin{array}{c}29.16 \pm 9.04 \\
(18-46)\end{array}$ & $\begin{array}{l}34.5 \pm 6.02 \\
(28-44)\end{array}$ & $\begin{array}{c}40.8 \pm 8.8 \\
(30-52)\end{array}$ \\
\hline GGT (U/L) & $\begin{array}{c}4.65 \pm 3.68 \\
(-1.2-5.7)\end{array}$ & $\begin{array}{l}9.66 \pm 2.21 \\
\quad(7-13)\end{array}$ & $\begin{array}{c}7.25 \pm 1.29 \\
(6-9)\end{array}$ & $\begin{array}{c}7.4 \pm 1.3 \\
(5-9)\end{array}$ \\
\hline ALT (U/L) & $\begin{array}{c}81.5 \pm 19.9^{\mathrm{a}} \\
(60-111)\end{array}$ & $\begin{array}{c}76.66 \pm 15.6^{a} \\
(50-102)\end{array}$ & $\begin{array}{c}67.5 \pm 6.02^{\mathrm{ab}} \\
\quad(58-74)\end{array}$ & $\begin{array}{c}32.2 \pm 12.35^{\mathrm{b}} \\
(9-44)\end{array}$ \\
\hline AST (U/L) & $\begin{array}{c}47.25 \pm 10.98 \\
(38-66)\end{array}$ & $\begin{array}{c}43.16 \pm 19.8 \\
(28-82)\end{array}$ & $\begin{array}{c}37.5 \pm 14.58 \\
(24-62)\end{array}$ & $\begin{array}{c}35.8 \pm 6.1 \\
(31-47)\end{array}$ \\
\hline $\mathrm{FA}(\mathrm{U} / \mathrm{L})$ & $\begin{array}{c}81.5 \pm 31.79^{a} \\
(298-386)\end{array}$ & $\begin{array}{c}101.83 \pm 25.11^{\mathrm{b}} \\
(74-148)\end{array}$ & $\begin{array}{c}125 \pm 10.51^{\mathrm{ab}} \\
(111-139)\end{array}$ & $\begin{array}{c}174.8 \pm 77.1^{\mathrm{ab}} \\
(103-290)\end{array}$ \\
\hline Phosphorus (mg/dL) & $\begin{array}{c}5.53 \pm 1.11 \\
(4-7.07)\end{array}$ & $\begin{array}{l}5.35 \pm 0.76 \\
(4.26-6.78)\end{array}$ & $\begin{array}{l}4.43 \pm 0.27 \\
(4.17-4.85)\end{array}$ & $\begin{array}{c}6.4 \pm 0.7 \\
(5.17-7.32)\end{array}$ \\
\hline Calcium (mg/dL) & $\begin{array}{c}11.24 \pm 0.88 \\
(10.35-12.45)\end{array}$ & $\begin{array}{c}9.49 \pm 0.79 \\
(8.53-10.48)\end{array}$ & $\begin{array}{c}10.98 \pm 0.69 \\
(10.02-11.86)\end{array}$ & $\begin{array}{c}9.56 \pm 1.33 \\
(8-11.89)\end{array}$ \\
\hline
\end{tabular}

Different letters on the line indicate that there is a statistical difference, with a $95 \%$ significance level.

In another study the authors observed lower percentage of eosinophils (average of 1) [4]. Nevertheless, in that case capybaras were bred for commercial purposes, confined for slaughter. In fact, Heijden et al. [8] compared white-blood cells profile of captive capybara groups and observed that those tick infested had significantly higher levels of circulating eosinophils in relation to those tick-free. Therefore, tick infestations are likely to induce eosinophilia in capybaras and may explain higher circulating proportion of these leukocytes in our study.

Much lower count of platelets and blood clotting of samples with anticoagulant in this work could be associated with some physiological features inherent to capybaras. Many attempts were required to obtain enough blood from each individual due to the rapid hemostasis. To obtain a good amount of blood free of clots, cannulating the vein with a large-gauge needle and let the blood drip into EDTA tubes was a better choice than the punction with a syringe or vacuum tubes systems. Another author reported problems with loss of blood material due to rapid clotting after blood collection by cardiac puncture into tubes with citrate in a slaughterhouse, after desensitization [12]. These issues may have contributed to the coagulation of samples from some animals and should be addressed in future studies with capybara blood.

A few significant differences were observed among capybara groups in the present work. Animals from P2 area $\left(1.83 \mathrm{~mm}^{3}\right)$ had significantly lower percentage of monocytes in relation to those from P3 $\left(6.0 \mathrm{~mm}^{3}\right)[P=0.0137]$. However global leucocyte counts did not differ between these 2 groups nor did the percentage of other leukocytes, therefore this difference may not have relevance for health status. Also, Kurloff cells were detected in 2 females in P1. This is not an unexpected observation since Kurllof cells can be found in peripheral blood of female rodents during follicular phase of estrous cycle [7].

Significantly low levels of blood albumin were observed between capybaras from P2 (mean $1.56 \mathrm{~g} / \mathrm{dL}$ ) compared to capybaras captured in P3 (mean $2.07 \mathrm{~g}$ / 
$\mathrm{dL}$ ) and P4 (mean $2.17 \mathrm{~g} / \mathrm{dL})[P=0.002]$. The values obtained in P3 and P4 were similar to those obtained from capybaras that live in the Pantanal (average of $2.5 \mathrm{~g} / \mathrm{dL}$ ), a natural environment for these rodents [2]. Other authors found similar albumin values (2.86 to $3.07 \mathrm{~g} / \mathrm{dL}$ ) in capybaras from a private park in Rio Grande do Sul [3]. Therefore, we speculate that the low albumin blood levels from capybaras captured in P2 can be attributed to a protein deficiency in the diet and related to the available food for those animals (mainly Lactuca sp.).

Free-living capybaras in pristine areas from Mato Grosso and Mato Grosso do Sul had an average blood alkaline phosphatase value of 171.5 U/L [2], close to that of capybaras captured in P4 (average 174.8 $\mathrm{U} / \mathrm{L}$ ) and higher than the values found for animals from P3 (average $125 \mathrm{U} / \mathrm{L}$ ), P2 (average $101.83 \mathrm{U} / \mathrm{L}$ ) and $\mathrm{P} 1$ (average $81.5 \mathrm{U} / \mathrm{L}$ ), with a significant difference between the values obtained between P2 and P1 $(P$ value $=0.0091)$. Once there were no other alterations in the red blood cells or hematocrit count, neither of phosphorus or calcium levels, the relationship of alkaline phosphatase with some systemic disease such as anemia or bone distress in the capybaras from our work seems unlikely. Since the offer of food diverged in each place, especially between capybaras from condominium (P1) and the privet market garden (P2), among others, the effect of the lack of nutrients in the diet upon alkaline phosphatase blood levels should be explored in forthcoming studies.

The ALT enzyme also showed statistical differences between P1 (81.5 U/L) and P2 (76.6 U/L) with $\mathrm{P} 4(32.2 \mathrm{U} / \mathrm{L})[P=0.0101]$. Because there was no GGT alterations, the ALT variation could be addressed to capture stress and not some previously hepatic condition.

Animals from P3 (34.3 mg/dL) and P4 (13.26 $\mathrm{mg} / \mathrm{dL})$ presented statistical difference for BUN $(P=$ 0.0086). Disparities in this renal enzyme can appear in animals with dehydration but this clinical parameter was not specifically addressed during sampling. Creatinine also showed statistical differences, between capybaras from P1 $(1.31 \mathrm{mg} / \mathrm{dL})$ and P2 $(0.74 \mathrm{mg} / \mathrm{dL})$ $[P=0.0028]$. Increased blood creatinine levels indicate kidney diseases. Nevertheless, such values were lower than the founds by others [18] in free-living capybaras from Pernambuco State $(1.51 \mathrm{mg} / \mathrm{dL})$. Overall, such differences rather indicate blood urea and creatinine fluctuations that are within physiological range of the species.

\section{CONCLUSION}

No major blood parameter differences between sex and age and among animal groups were detected in capybaras free-living within urban areas of a major city. At the same time some parameters differed in relation to those of literature describing either captive animals or free-living under different environment. This work contributes to the standardization of free-living capybaras' physiological parameters in urban areas.

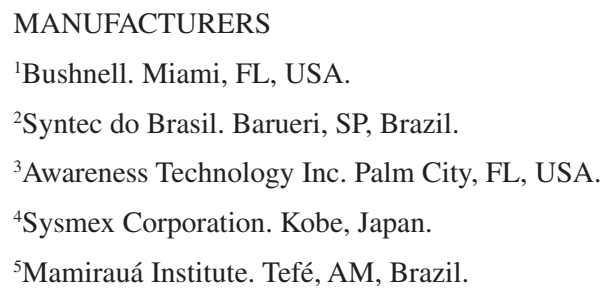

Funding. CAPES, FAPEMIG and FAPERJ.

Ethical approval. This study was approved by the Ethics Committee on the Use of Animals (CEUA Universidade Federal Fluminense) protocol number - 1010. Authorization for capybara capture and handling was given by the Brazilian Biodiversity Information and Authorization System (SISBio) permission number 60590. Access to parks and public places for animal capture was authorized by the City Hall of Uberlândia.

Declaration of interest. The authors report no conflicts of interest. The authors alone are responsible for the content and writing of paper.

\section{REFERENCES}

1 Alho C.J.R. \& Rondon N.L. 1987. Habitats, population densities, and social structure of capybaras (Hydrochaeris hydrochaeris, rodentia) in the Pantanal, Brazil. Revista Brasileira de Zoologia. 4(2): 139-149.

2 Benatti H.R. 2020. Comparison of morphometric patterns and blood biochemistry in capybaras (Hydrochoerus hydrochaeris) of human-modified landscapes and natural landscapes. 90f. São Paulo, SP. Tese (Doutorado em Ciências) - Programa de Pós-Graduação em Epidemiologia Experimental Aplicada à Zoonoses e Saúde Animal, Universidade São Paulo. 
3 Bernal L., Feser M., Martínez-Subiela S., García-Martínez J.D., Cerón J.J. \& Tecles F. 2011. Acute phase protein response in the capybara (Hydrochoerus hydrochaeris). Journal of Wildlife Diseases. 47(4): 829-835.

4 Corredor-Matus J.R. \& Rodrígues-Pulido J. 2010. Estudio del perfil hemático y metabólico de chigüiros (Hydrochaeris hydrochaeris) (LINNAEUS, 1766) em confinamento. Orinoquia. 14(1): 95-109.

5 Di Chiacchio R.G.M. 2012. Avaliação sanitária de capivaras (Hydrochoerus hydrochaeris) de vida livre presentes na região da Cantareira - zona norte de São Paulo. 125f. São Paulo, SP. Dissertação (Mestrado em Ciências). Programa de Pós-Graduação em Patologia. Experimental e Comparada São Paulo: Programa de Pós-Graduação em Patologia Experimental e Comparada, Universidade de São Paulo.

6 Ferraz K.M.P.M.B., Ferraz S.F.B., Moreira J.R., Couto H.T.Z. \& Verdade L.M. 2007. Capybara (Hidrochoerus hydrochaeris) distribution in agroecosystems: a cross-scale habitat analysis. Journal of Biogeography. 34: 223-230.

7 Hamouzova P., Cizek P., Jekl V., Gozdziewska-Harlajczuk K. \& Kleckowska-Nawrot J. 2021. Mast cells and Kurloff cells - Their detection throughout the oestrous cycle in normal guinea pig ovaries and in guinea pigs with cystic rete ovarii. Research in Veterinary Science. 136: 512-518.

8 Heijden K.M., Szabó M.J.P., Matushima E.R., Veiga M.L., Santos A.A. \& Egami M.I. 2003. Valores Hematológicos e identificação morfo-citométrica de células sanguíneas de capivaras (Hydrochoerus hydrochoeris) parasitadas por carrapatos e capivaras livres de infestação. Acta Scientiarum Animal Sciences. 25(1): 143-150.

9 Instituto Brasileiro do Meio Ambiente (IBAMA). 2006. Diagnóstico populacional da capivara. 8p. Ministério Brasileiro do Meio Ambiente. Available at <http://www.compras.mg.gov.br/images/stories/arquivoslicitacoes/2017/ SEPLAG/15.09.2017/gpfaf-tr-09-capivaras-ibama-diagnostico-populacional-da-capivara.pdf>.

10 Intituto Brasileiro de Geografia e Estatística (IBGE). 2018. Censo populacional. Available at $<$ https://cidades.ibge. gov.br>.

11 Labruna M.B. 2013. Brazilian spotted fever: the role of capybaras. In: Moreira J.R., Ferraz K.M.P.M.B., Herrera E.A. \& Macdonald D.W. (Eds). Capybara: Biology, use and Conservation of an Exceptional Neotropical Species. New York: Springer Science Business Media, pp.371-383.

12 Leitão D.P.S., Polizello A.C.M. \& Rothschild Z. 2000. Coagulation and fibrinolysis in capybara (Hidrochaeris hydrochaeris), a close relative of the guinea-pig (Cavia porcellus). Comparative Biochemistry and Physiology. 125: 113-120.

13 Luz H.R., Costa F.B., Benatti H.R., Ramos V.N., Serpa M.C.A., Martins T.F., Costa I.C.L.A., Ramirez D.G., Muñoz-Leal S., Ramirez-Hernandes A., Binder L.C., Carvalho M.P., Rocha V., Dias T.C., Simeoni C.L., Brites Neto J., Brasil J., Nievas A.M., Monticelli P.F., Moro M.E.G., Lopes B., Aguiar D.M., Pacheco R.C., Souza C.E., Piovezan U., Juliano R., Ferraz K.M.P.M.B., Szabó M.J.P. \& Labruna M.B. 2019. Epidemiology of capybaraassociated Brazilian spotted fever. Plos Negleted Tropical Diseases. 13(9): 1-24.

14 Pajuaba-Neto A.A., Limongi J.E., Silva V.P. \& Szabó M.P.J. 2019. Knowledge, atitudes and practices about capybara in anthropic areas: perception of society. Revista Brasileira de Geografia Médica e da Saúde (Hygeia). 15(34): 35-52.

15 Pascoli G.V. 2014. Carrapatos e riquétsias em parque urbano de Uberlândia, Minas Gerais: ecologia e biodiversidade associadas. 160f. Uberlândia, MG. Tese (Doutorado em Biologia) - Programa de Pós-Graduação em Ecologia e Conservação do Recursos Naturais, Universidade Federal de Uberlândia.

16 Queirogas V.L., Del Claro K., Nascimento A.R.T. \& Szabó M.P.J. 2012. Capybaras and ticks in the urban areas of Uberlândia, Minas Gerais, Brazil: ecological aspects for the epidemiology of tick-borne diseases. Experimental and Applied Acarology. 57: 75-82.

17 SEPLAN. 2019. Secretaria Municipal de Planejamento Urbano - Núcleo de Pesquisa Estatística e Banco de Dados. Banco de Dados Integrados de Uberlândia - DBI. Available at <https://www.uberlandia.mg.gov.br/prefeitura/secretarias/ planejamento-urbano/servicos-online/>.

18 Souza D.S., Yang S. G.N.S., Alves A.C.A., Pontes R.M., Carvalho C.C.D., Soares P. C. \& Oliveira J.B. 2020. Perfil hematológico e bioquímico sérico de capivaras (Hydrochoerus hydrochaeris) de vida livre nos biomas Mata Atlântica e Caatinga. Arquivos Brasileiros de Medicina Veterinária e Zootecnia. 72(2): 461-470. 